Publié dans "Quaternaire, 19(3), 2008, 217-228,

qui doit être cité pour toute référence à cet

article

\title{
UNE NAPPE ALLUVIALE ÉTAGÉE DU PLÉISTOCÈNE SUPÉRIEUR DANS LA VALLÉE DE DELÉMONT (JURA SUISSE) : LITHOSTRATIGRAPHIE ET DATATION
}

\author{
Luc BRAILLARD \& Michel GUÉLAT
}

RÉSUMÉ

La vallée de Delémont, dans le Jura suisse, est caractérisée par un système de trois nappes alluviales étagées, T1, T2 et T3, mises en place en contexte périglaciaire. Cet article présente la stratigraphie détaillée de la plus haute de ces terrasses, T3, correspondant à des graviers fluviatiles revêtus par des dépôts limoneux qui montrent un horizon fragipan et des polygones de gel à leur sommet. Des datations par luminescence (OSL) y ont été réalisées. Les résultats de ces dernières, qui s'insèrent dans les Pléniglaciaires moyen et supérieur würmiens, sont présentés en détail et discutés.

Mots-clés : nappes alluviales, lœess, fragipan, datation OSL, Pléistocène supérieur, Jura.

\section{ABSTRACT}

AN UPPER PLEISTOCENE STEPPED ALLUVIAL SHEET IN DELÉMONT VALLEY (SWISS JURA): LITHOSTRATIGRAPHY AND DATING Located in the Swiss Jura, the Delémont basin is characterized by a system of three stepped alluvial sheets, T1, T2 and T3, deposited in a periglacial environment. This paper presents the detailed stratigraphy of the highest of these terraces, T3, that consists of fluviatile gravels covered by silty deposits, including a fragipan horizon, and frost polygons at their top. The results of optically stimulated luminescence dating (OSL), pointing to the Würmian Middle to Upper Pleniglacials, are presented in detail and discussed.

Keys-words: alluvial sheets, loess, fragipan, OSL dating, Late Pleistocene, Jura.

\section{1 - INTRODUCTION}

Les nappes alluviales étagées présentent un intérêt qui n'est plus à démontrer, aussi bien en termes de variations climatiques et de processus néotectoniques que pour la reconstitution environnementale et le calage chronologique des gisements archéologiques.

Une telle succession de terrasses fluviatiles s'inscrit dans le paysage du bassin de Delémont, vaste synclinal situé dans la partie septentrionale de la chaîne jurassienne, côté suisse (fig. 1). Ce système exprime de manière éloquente la sédimentation en contexte périglaciaire, cette vallée se positionnant en dehors - mais à proximité - des zones englacées au cours du Pléistocène (Aubry et al., 2005).

Engendrées par la construction d'une autoroute, d'importantes opérations de sauvetage archéologique ont été accomplies dans cette région; des études approfondies $\mathrm{du}$ contexte géologique, et tout particulièrement des formations quaternaires, font partie intégrante de ces travaux. En plus des informations stratigraphiques et génétiques, de nombreuses données chronologiques ont été acquises par datations radiocarbone, mais aussi par la palynologie ou la typologie du mobilier archéologique. Cependant, les nappes alluviales contiennent rarement les matières nécessaires à ce type de datations. La luminescence stimulée optiquement (OSL) offre à point nommé la possibilité de déterminer l'âge des formations fluviatiles: confrontés aux données géomorphologiques et lithostratigraphiques, les résultats fournis par cette méthode permettent de préciser les schémas chronologiques. Le présent article concerne un aspect précis de cette problématique, à savoir la genèse et la datation de la plus haute de ces nappes dominant la plaine actuelle. Bien que très ciblée, cette approche a des implications évidentes pour l'histoire sédimentaire du Quaternaire à l'échelle régionale.

\section{1 - TRAVAUX ET MÉTHODES}

Menées depuis une vingtaine d'années, les recherches liées à l'autoroute A16 - dite Transjurane - ont permis d'acquérir une vision renouvelée du sous-sol dans le 


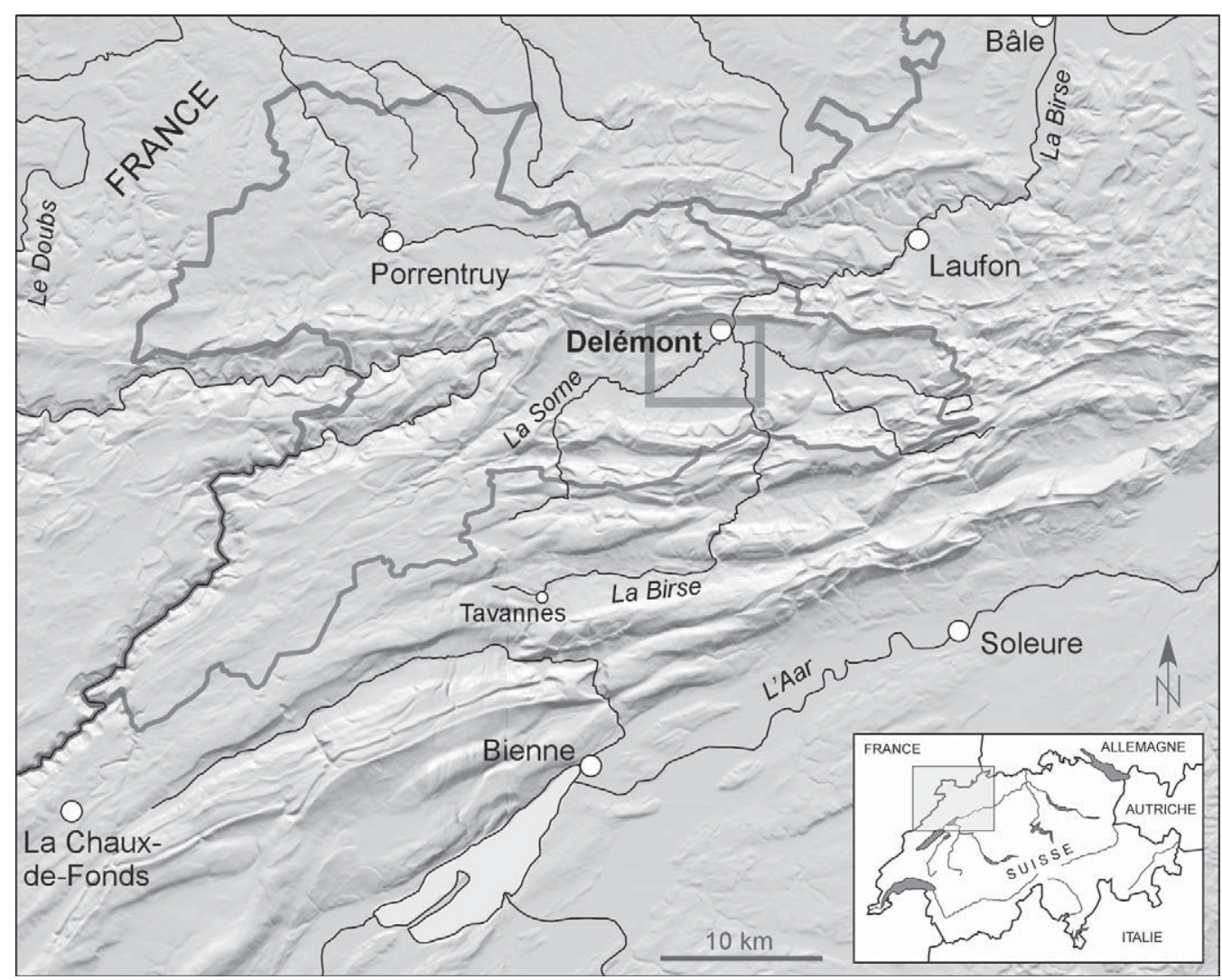

Fig. 1 : Situation géographique. Le cadre correspond à la figure 2. Reproduit avec l'autorisation de swisstopo (BA071520). Fig. 1: Geographical location. Frame corresponds to figure 2. Reproduced with permission of swisstopo (BA071520).

canton du Jura, entité à laquelle appartient la vallée de Delémont. La prospection systématique du tracé autoroutier (15 km de long sur $35 \mathrm{~m}$ de large pour la vallée de Delémont), le suivi des fouilles archéologiques (une vingtaine de sites d'importance inégale fouillés entre 1988 et 2005), ainsi que des forages complémentaires ont permis une approche environnementale de la plupart des milieux naturels de la région tels que plaines alluviales, versants et plateaux. Ces études ont aboutit à plusieurs publications sur le Quaternaire et la colonisation humaine de la vallée (Guélat et al., 1993; Braillard et al., 2002; Guélat, 2006a). Cependant, les résultats concernant la zone centrale du bassin, intensivement investiguée sur le terrain (Braillard \& Guélat, 2000, 2002) et sur laquelle se focalise le présent article, sont encore largement inédits.

Les terrains quaternaires de la vallée de Delémont ont, au XIX ${ }^{\mathrm{e}}$ siècle déjà, fait l'objet d'une description assortie d'une insertion stratigraphique (Greppin, 1855). Avec la publication, au début du Xx $\mathrm{X}^{\mathrm{e}}$ siècle, de la première feuille de l'Atlas géologique de la Suisse, les différentes nappes alluviales perchées sont individualisées (Liniger, 1925). Plus récemment, une étude géomorphologique propose une insertion chronologique de ces terrasses en tentant de les raccorder avec celles du Rhin, via la vallée de la Birse (Barsch, 1969). Essentiellement basée sur des arguments topographiques, cette identification néglige certains aspects de la problématique, en particulier ceux ayant trait à la lithologie.

Seule une caractérisation obtenue par sondages et analyses permet en effet de saisir toute la complexité d'une telle succession de nappes étagées, à savoir leur nature, leur relation avec la roche encaissante et, par déduction, leur genèse et leur âge relatif.

Des analyses sédimentologiques ont été effectuées dans cette intention au Laboratoire de l'IPNA (Institut für Prähistorische und Naturwissenschaftliche Archäologie) de l'Université de Bâle sur des échantillons bien ciblés, provenant de profils ouverts dans les différentes unités alluviales. La granulométrie des fractions graveleuses $(>2 \mathrm{~mm})$ a été effectuée par tamisage à sec. Les fractions sableuses (de 63 microns à $2 \mathrm{~mm}$ ) ont subi un tamisage humide sur colonne vibrante (laborette Fritsch), selon le procédé classique. La fraction silto-argileuse (<63 microns) a été analysée à l'aide d'un granulomètre laser (Malvern MasterSizer MS20); précisons que cet appareillage ne permet pas d'analyser les fractions sableuses supérieures à $1 \mathrm{~mm}$.

Pour le traitement informatique des résultats bruts, une application du logiciel Excel a été développée. La construction de la courbe granulométrique s'effectue par calcul proportionnel, soit, pour chaque fraction, un rapport au poids total de l'échantillon analysé. Nous sommes conscients du fait que les résultats du granulomètre laser sont exprimés en pourcentages volumiques et peuvent de ce fait différer de ceux issus d'une méthode pondérale. Une série de tests a en effet montré que la teneur en argiles ( $<2$ microns) a tendance à être sousestimée par la mesure au laser, en particulier pour les échantillons riches en fines (Braillard, 2006). Dans la pratique, cette manière de faire a toutefois fait ses preuves: l'erreur engendrée par l'utilisation de deux 
méthodes distinctes pour construire une seule et même courbe est si faible - sédiments très argileux exceptés qu'elle n'a pour ainsi dire aucune influence sur le diagnostic génétique déduit de celle-ci, ce qui au final constitue le but de la démarche.

Des tests géochimiques ont également été réalisés sur ces mêmes échantillons; pratiqués en routine à l'IPNA, ceux-ci comprennent le taux total de carbonates (mesuré à l'aide d'un calcimètre de Müller), la teneur en matière organique (par perte au feu résultant d'une combustion à $\left.1000^{\circ} \mathrm{C}\right)$, la teneur totale en phosphates et en humus (obtenue par colorimétrie) et le $\mathrm{pH}(\mathrm{pH} \mathrm{KCl})$. En complément, une analyse microscopique a été rendue possible grâce à l'induration (réalisée également à l'IPNA) d'échantillons de sédiments meubles, issus de profils distincts, à partir desquels des lames minces ont été fabriquées par Th. Beckmann, à Braunschweig, Allemagne (laboratoire indépendant).

Des datations par luminescence stimulée optiquement (OSL) ont été effectuées par le laboratoire du Département de Géographie de l'Université de Cologne. La méthode de prélèvement, sur le terrain, a consisté a planter horizontalement des tubes opaques en PVC (diamètre: $5 \mathrm{~cm}$; longueur: $30 \mathrm{~cm}$ ) dans le profil stratigraphique du sondage OSL 1, afin de pouvoir prélever du sédiment non perturbé et surtout non contaminé par la lumière. Les extrémités des tubes extraits du profil, partiellement exposées à la lumière, ont été sciées et utilisées pour le dosage des radionucléides (uranium, thorium et potassium) par activation neutronique réalisée aux Laboratoires Becquerel de Sydney, Australie. La partie centrale des tubes, plus précisément la fraction granulométrique 4-11 microns du sédiment, a été utilisée pour déterminer les paléodoses selon la méthode régénérative sur aliquotes multiples (Wintle, 1997).

\section{2 - LES NAPPES ALLUVIALES ÉTAGÉES DANS LA VALLÉE DE DELÉMONT}

\section{1 - CONTEXTE GÉOLOGIQUE ET HYDROGRAPHIE}

Correspondant à un large synclinal à fond plat, la vallée de Delémont s'étire sur $25 \mathrm{~km}$ selon un axe ouestest, pour une largeur maximale de $8 \mathrm{~km}$, totalisant une superficie de $200 \mathrm{~km}^{2}$ environ. Situé au nord-ouest de la Suisse, ce bassin se trouve au coeur du Jura plissé, à midistance entre le Fossé rhénan et le Plateau suisse dont il est séparé d'une vingtaine de kilomètres à vol d'oiseau (fig. 1). Délimité par des anticlinaux de calcaires et marnes du Jurassique, son fond s'étend à une altitude moyenne de $450 \mathrm{~m}$; il est revêtu de molasse oligocène et, plus localement, de cailloutis vosgiens miocènes. Les dépôts du Quaternaire consistent essentiellement en des formations fluviatiles dont l'épaisseur atteint 3 à $5 \mathrm{~m}$.

Le secteur pris en compte pour cette approche couvre environ $10 \mathrm{~km}^{2}$ au centre même du bassin, directement au sud de la petite ville de Delémont (fig. 2). L'étagement des nappes alluviales quaternaires s'y marque de manière explicite dans la zone de confluence entre deux rivières. Il s'agit d'une part de la Birse, cours d'eau principal en provenance du sud et qui s'écoule vers le nord,

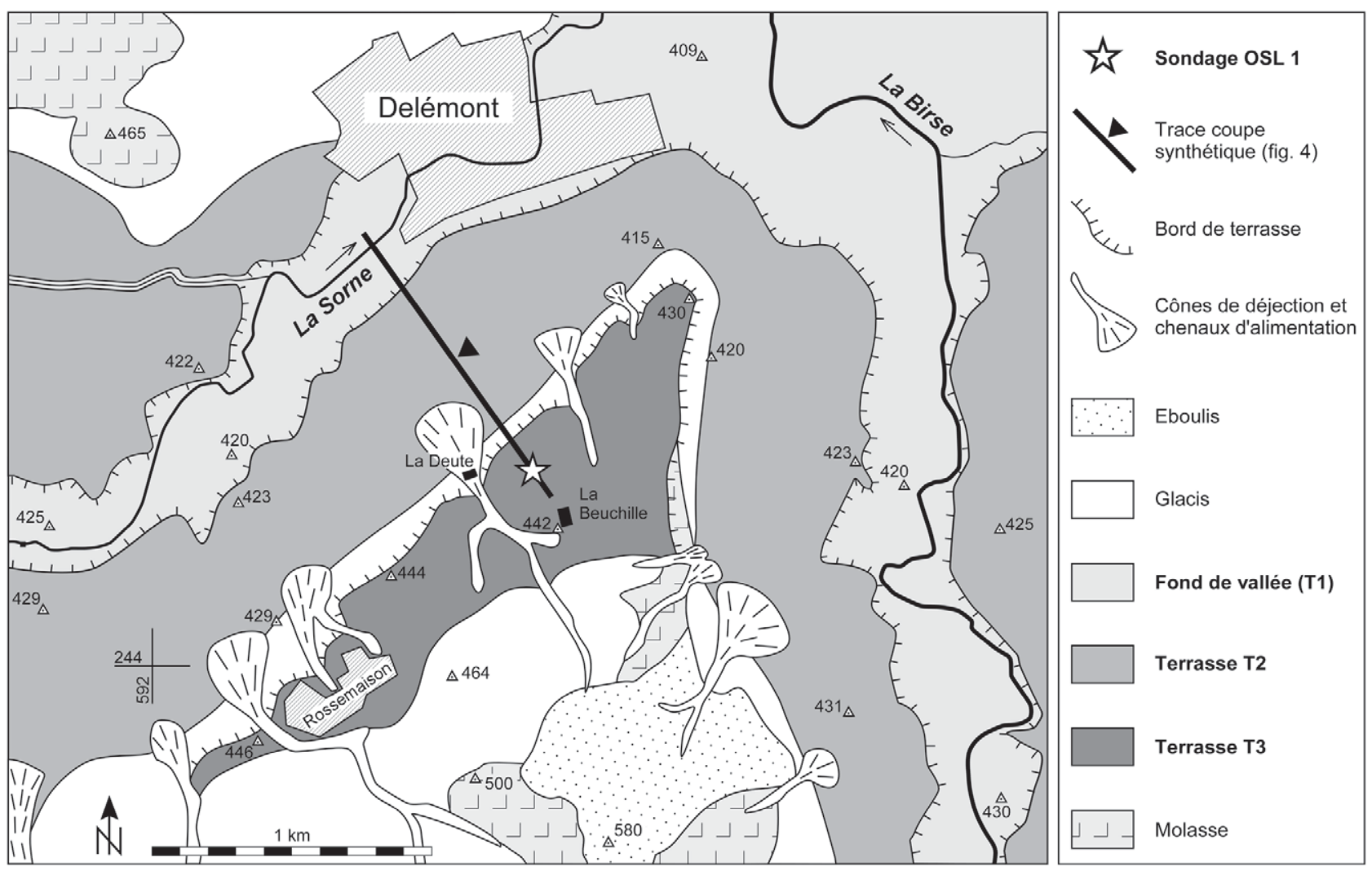

Fig. 2 : Carte géomorphologique des terrasses fluviatiles au sud de Delémont (modifié d'après Barsch, 1969 et Keller \& Liniger, 1930).

Fig. 2: Geomorphological map of the fluviatile terraces south of Delémont (modified after Barsch, 1969 and Keller \& Liniger, 1930). 
jusqu'à Bâle, pour rejoindre le Rhin; et d'autre part de la Sorne, affluent de la précédente, en provenance de la partie occidentale du bassin. Logées en fond de vallée, à une altitude comprise entre $410 \mathrm{~m}$ et $420 \mathrm{~m}$, ces deux rivières sont séparées par le Montchaibeux, colline formée de molasse dont le sommet culmine à $630 \mathrm{~m}$.

\section{2 - LA DERNIÈRE GLACIATION ET LES LCESS}

A l'instar du territoire du canton du Jura, la vallée de Delémont n'a pas été envahie par les glaciers au Pléistocène, bien qu'elle en ait subit l'influence vu leur relative proximité. Autrement dit, cette région peut se définir comme un domaine périglaciaire caractérisé par des édifices sédimentaires assez originaux pour la Suisse. Les moraines recensées les plus proches ne se rencontrent qu'à une douzaine de kilomètres plus au sud, dans la vallée de Tavannes, au nord de la ville de Bienne (Pfirter et al., 1997).

Il y a 21000 ans environ, période durant laquelle le maximum d'extension glaciaire würmien aurait été atteint sur le plateau suisse (Ivy-Ochs et al., 2004), l'inlandsis alpin n'a en effet pas franchi les premiers plis de la chaîne bordant le Plateau suisse (fig. 3), tandis qu'au sud-ouest, la calotte jurassienne ne s'étendait guère au-delà de La Chaux-de-Fonds (Campy \& Arn, 1991). Quant aux glaciers générés par les massifs des Vosges et de la Forêt-
Noire, plutôt modestes, ils n'ont jamais pu atteindre le Jura (Monjuvent \& Nicoud, 2002). Outre la limite de l'extension des glaces lors du maximum würmien, la figure 3 montre l'aire de répartition des loess, particulièrement développés dans le Fossé rhénan (18 m à Achenheim $(\mathrm{F})$, Sommé et al., 1986; 17 m à Nussloch (D), Antoine et al., 2002). Côté suisse, des séquences éoliennes d'importance ont été identifiées dans la région bâloise (Zollinger, 1991), mais également en Ajoie, près de Porrentruy, où elles n'ont qu'un à deux mètres d'épaisseur pour le Pléistocène supérieur (Aubry et al., 2000; Braillard, 2006).

La sédimentation lossique a également «saupoudré» le Jura plissé comme l'ont montré des travaux menés sur les sols jurassiens, mais au sud-ouest de la zone d'étude, en territoires vaudois et neuchâtelois (Pochon, 1973; Havlicek \& Gobat, 1996). Fait nouveau détaillé dans le présent article, des limons d'origine éolienne ont également été mis en évidence dans la vallée de Delémont, où ils revêtent l'unité alluviale la plus élevée dans le paysage. Cette présence avait déjà été suspectée par Barsch (1969), alors que Liniger (1925) dit explicitement qu'il n'a pas pu constater l'existence de lœss quaternaires dans cette région.

\section{3 - LES NAPPES FLUVIATILES : PRÉSENTATION}

Au cours du Quaternaire, dans la partie centrale du bassin de Delémont, la Sorne et la Birse ont façonné le

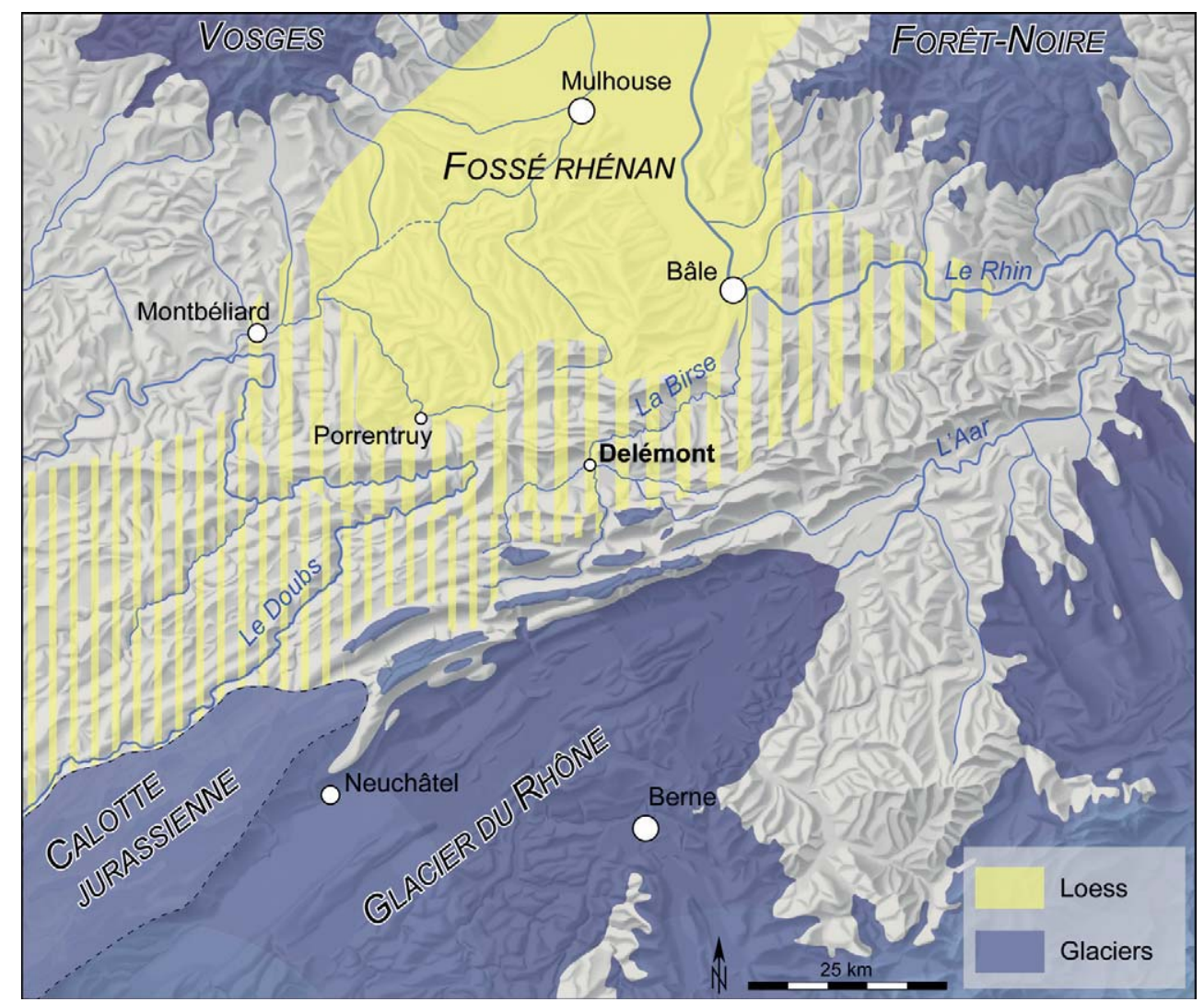

Fig. 3 : Extension des glaces durant le maximum glaciaire du Würm dans le NW de la Suisse, il y a environ 21000 ans. Reliefs et limites des glaces (glaciers du Rhône, des Vosges et de la Forêt Noire) tirés de l'Atlas de la Suisse version 2.0, 2005; terminaison du glacier de calotte jurassien tiré de Campy \& Arn 1991. Reproduit avec l'autorisation de swisstopo (BA071520).

Fig. 3: Ice cover during the würmian glacial maximum in northwestern Switzerland, about 21 000 years ago. Topography and ice cover limits (Rhône, Vosges and Black Forest glaciers) after Atlas of Switzerland 2.0, 2005 ; extension of the Jura ice sheet after Campy \& Arn 1991. Reproduced with permission of swisstopo (BA071520). 
relief en plaquant sur la molasse oligocène un système de trois nappes alluviales étagées dénommées T3, T2 et T1 (fig. 2 et 4).

La plus élevée et ancienne, l'unité T3, s'appuie sur le flanc nord-ouest du Montchaibeux, à une altitude moyenne comprise entre 445 et $430 \mathrm{~m}$. Elle surmonte les unités $\mathrm{T} 2$ et $\mathrm{T} 1$ respectivement d'une quinzaine et d'une vingtaine de mètres. Elle détermine une plate-forme topographique d'une superficie assez restreinte (environ 90 hectares) appelée plateau de la Beuchille, du nom de la ferme exploitant ce replat qui se prolonge jusqu'au village de Rossemaison, au sud-ouest. Cette nappe a été reconnue par Liniger (1925) qui parle de Schotterterrasse (soit littéralement "graviers en terrasse»); Barsch (1969), par analogie intentionnelle avec la région bâloise, la dénomme Hochterrasse (soit «haute terrasse») et l'insère chronologiquement dans l'avant-dernière glaciation («Riss »). lambeaux d'altérites plaqués sur la molasse, à une altitude supérieure dans le versant. Ceux-ci n'ont pas pu être datés, mais leur position topographiquement élevée ainsi que le fort degré d'altération semblent indiquer qu'il s'agit des restes d'une terrasse fluviatile antérieure à T3.

La deuxième nappe alluviale, l'unité $\mathrm{T} 2$, se situe au pied des versants et se caractérise, contrairement à la précédente, par une assez grande extension latérale. On la rencontre en effet dans la totalité du bassin de Delémont, aux abords du fond de vallée actuel revêtu par l'unité $\mathrm{T} 1$, dont elle se distingue cependant par un étagement de quelques mètres. Qualifiée de «basse terrasse principale» (Hauptniederterrasse) et attribuée au Würm par Barsch (1969), sa surface s'établit à une altitude allant de 430 à $415 \mathrm{~m}$ dans le secteur considéré. La nappe alluviale T2 peut localement atteindre $6 \mathrm{~m}$ d'épaisseur, comme on peut le constater dans les gravières exploitées

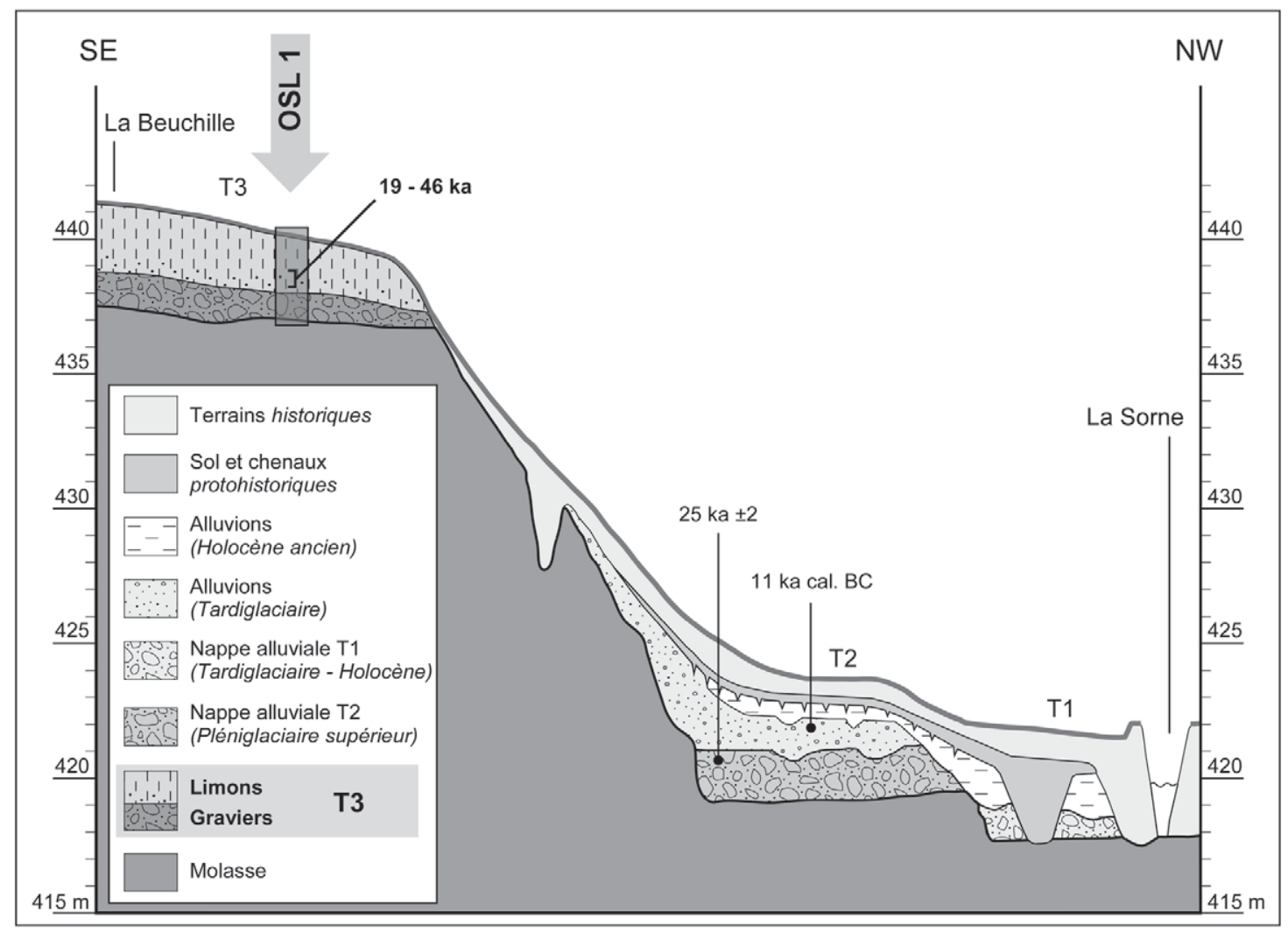

Fig. 4 : Coupe synthétique à travers la vallée de Delémont (échelle verticale exagérée 10x) et position du sondage OSL 1 sur la terrasse T3. Situation de la coupe : voir fig. 2.

Fig. 4: Synthetic cross-section through the Delémont basin (vertical exaggeration 10x) with profile OSL 1 on terrace T3. Cross-section situation: see fig. 2.

Selon nos observations, l'unité T3 correspond à des graviers fluviatiles revêtus par des dépôts limoneux qui montrent des polygones de gel à leur sommet. Dans un sondage creusé à la pelle mécanique à proximité de la ferme de la Beuchille (sondage OSL 1, fig. 5), un profil complet de ces dépôts a été levé. Des prélèvements y ont été effectués pour des analyses sédimentologiques et des datations OSL, dont les résultats sont exposés en détail plus bas (chap. 3). Les graviers de la nappe alluviale T3 constituent la plus ancienne formation quaternaire rencontrée dans le secteur d'étude, si l'on excepte des près de la Birse. Il s'agit d'un cailloutis riche en sables et à stratification oblique, mis en place selon un style fluvial en tresse; des déformations dues à l'action du gel ont été observées à sa base. Selon des arguments lithologiques et stratigraphiques, ce cailloutis s'est déposé au cours d'un épisode würmien froid, vraisemblablement le Pléniglaciaire supérieur. Ceci a été confirmé par une datation OSL, effectuée dans des niveaux sableux de cette unité, qui a livré un âge de $25 \pm 2$ ka (Guélat, 2006b). D'autres éléments chronologiques obtenus à l'ouest de Delémont indiquent que la mise en place de 


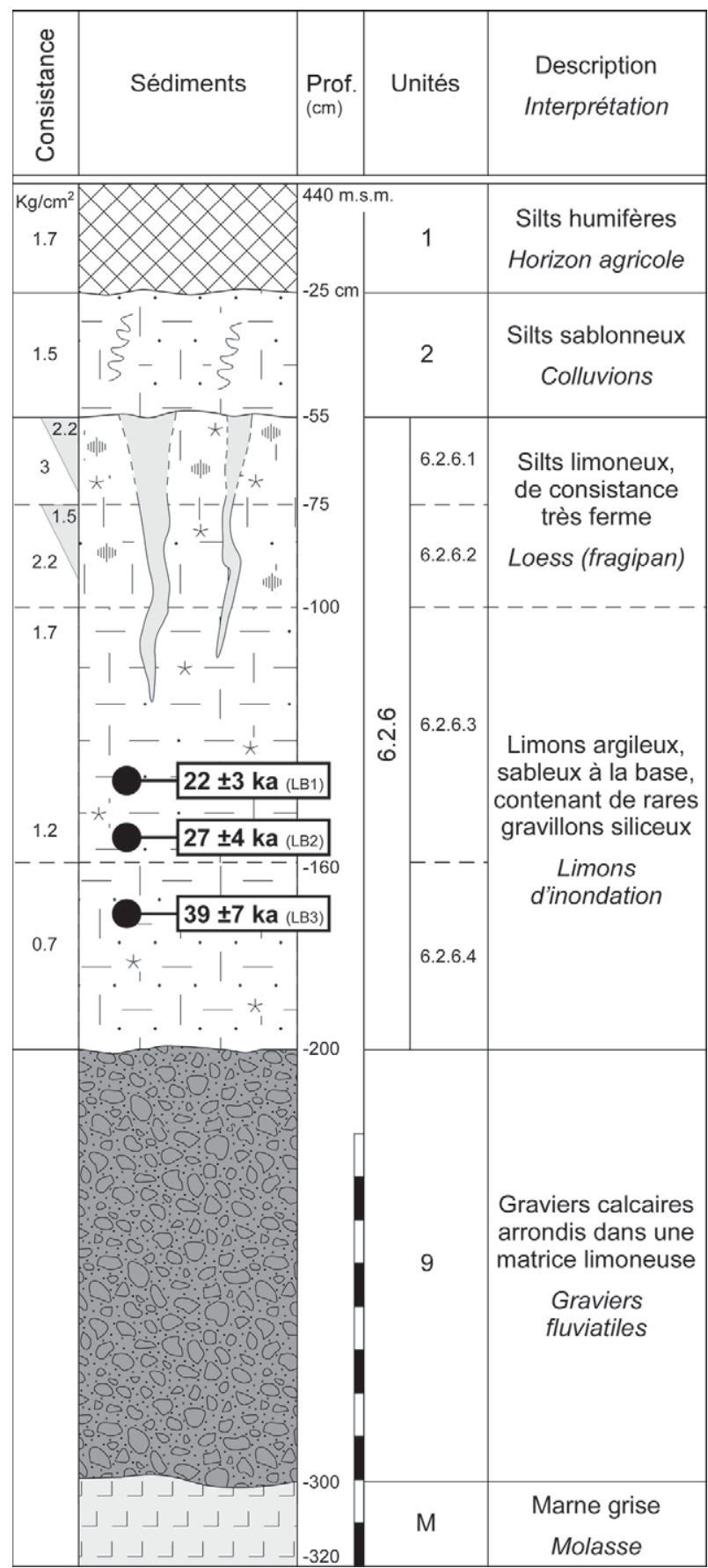

Fig. 5: Stratigraphie du sondage OSL 1, représentatif de T3, et résultats des datations par luminescence.

Fig. 5: Stratigraphy of the profile OSL 1, representative of T3, and results of the luminescence dating.

cette formation fluviatile s'est poursuivie jusque vers 20 ka BP (Guélat, 2006a).

La troisième nappe alluviale individualisée, T1, occupe la position géomorphologique la plus basse, en fond de vallée. Dans les vallons latéraux, elle se superpose au cailloutis pléniglaciaire T2 avec lequel elle se confond parfois. Ressemblant en effet à ce dernier du point de vue faciès, l'unité T1 est formée de graviers dont l'épaisseur varie entre 2 et $4 \mathrm{~m}$. Siège de la nappe phréatique, ces alluvions grossières sont fréquemment recouvertes par d'épais dépôts holocènes. Vu ces condi- tions techniques difficiles, aucune datation n'est à ce jour disponible pour ce cailloutis; par déduction stratigraphique, on peut toutefois avancer que sa mise en place a eu lieu durant le Tardiglaciaire.

Reposant généralement sur l'unité T2, une séquence se rapportant au Tardiglaciaire est localement préservée, en particulier au débouché des vallées latérales, sous forme de petits cônes de déjection. Pouvant atteindre $2 \mathrm{~m}$ d'épaisseur, ces formations passent latéralement à des dépôts alluviaux renfermant des charbons de bois ayant livré par ${ }^{14} \mathrm{C}$ un âge proche de 11000 cal. BC (Guélat, 2005; Guélat, à paraître). La métamorphose fluviale, à savoir le passage du type tressage au type méandrage, s'est quant à elle opérée dans la vallée de Delémont au cours de l'interstade Bølling (Guélat et al., 1993).

Avec la persistance du système méandrisant et une migration latérale des cours d'eau plutôt limitée, l'Holocène a vu, pour sa majeure partie, d'assez faibles taux de sédimentation. En coupe, cela s'exprime par une troncature progressive des dépôts tardiglaciaires laissant place à des alluvions chenalisées, elles-mêmes recoupées par des séquences plus tardives. Celles-ci se rapportent souvent à la période protohistorique, avec laquelle semble débuter un net accroissement de la sédimentation alluviale. Contemporaine de la première réelle colonisation humaine de la région, cette époque se marque également par un sol fossile apparu à maints endroits sur le tracé de la Transjurane (Braillard et al., 2002). Quant aux dépôts des temps historiques, ils sont particulièrement bien développés dans la vallée de Delémont (Guélat et $a l$. , à paraître).

\section{3 - LA NAPPE ALLUVIALE T3 : STRATIGRAPHIE ET DATATION}

\section{1 - LITHOSTRATIGRAPHIE}

L'ouverture sur le plateau de la Beuchille de plus de 150 sondages à la pelle mécanique, approfondis pour la plupart jusqu'au toit de la molasse tertiaire, a permis de construire trois coupes synthétiques détaillées qui traversent, d'ouest en est, la totalité de la nappe alluviale T3 (Braillard \& Guélat, 2002; Braillard, à paraître). Celle-ci totalise une puissance maximale de $4 \mathrm{~m}$. Les graviers fluviatiles qui constituent sa base (unité 9) sont coiffés par des dépôts limoneux, décarbonatés (unités 6.2.6, 6.2.5, 6.1 et 2, fig. 5). La géométrie des dépôts, acquise à l'occasion de cette campagne de sondages, a en outre pu être confirmée lors du creusement de la tranchée autoroutière qui traverse le plateau de la Beuchille (fig. 6).

\subsection{1 - Unité 9}

Il s'agit de graviers calcaires arrondis, moyennement classés, dans une matrice grise carbonatée, tantôt limoneuse, tantôt sableuse. Des éléments de la classe granulométrique des cailloux et des blocs sont également présents. Assez rares, ces derniers sont subarrondis à subanguleux et peuvent atteindre $45 \mathrm{~cm}$ de diamètre. Une petite fraction des éléments $(<10 \%)$ est constituée 


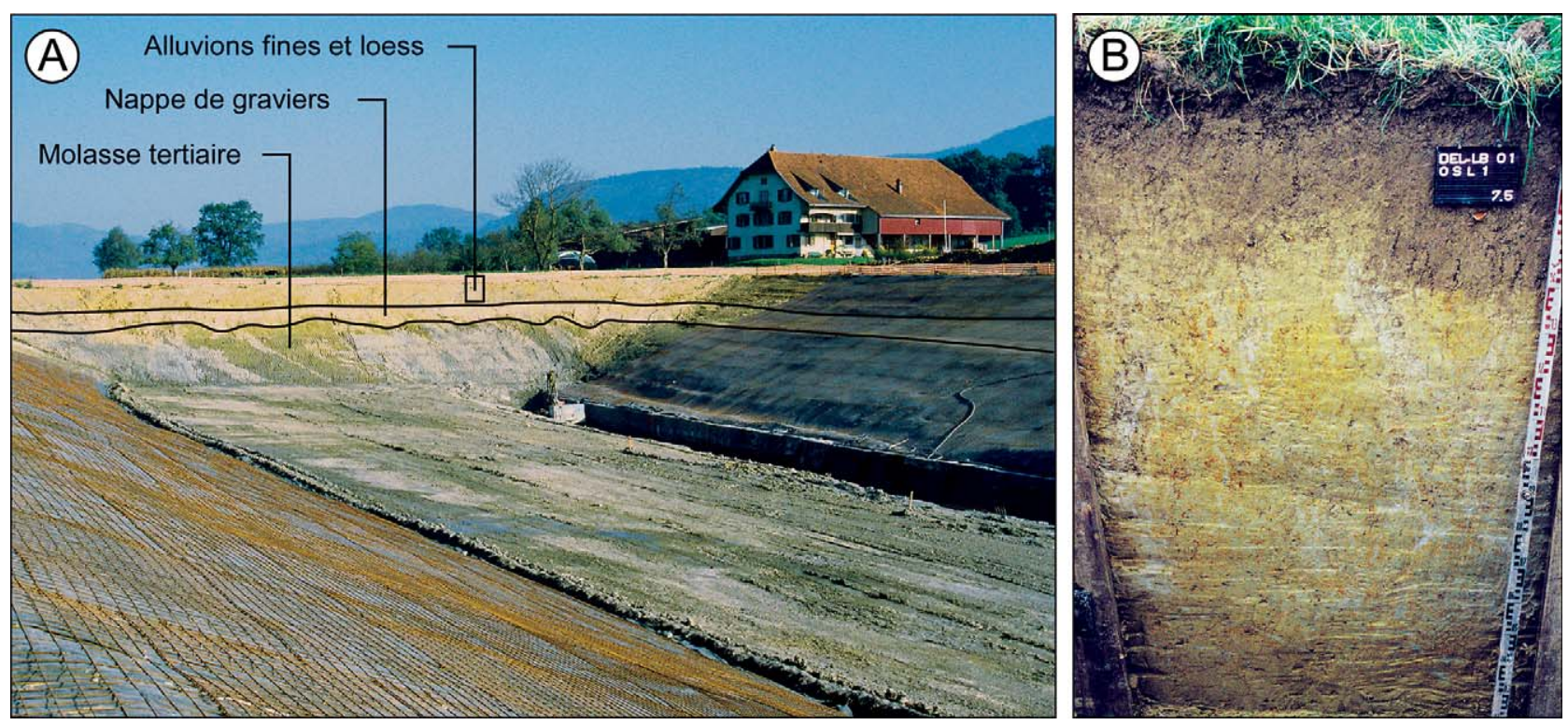

Fig. 6: (A) Vue en coupe de la nappe alluviale T3 près de la ferme de la Beuchille, au sud de Delémont et (B) détail de la partie sommitale constituée d'alluvions fines et de loess dans lesquels s'est développé un fragipan.

Fig. 6: (A) Alluvial sheet T3 as seen in cross-section near the farm of la Beuchille, south of Delémont, and (B) detail of its uppermost part made of fine alluvium and loess in which a fragipan developed.

de graviers siliceux. La base de cette unité est nettement érosive et des figures de déformation dues au gel y ont été localement observées. Le sommet des graviers, au contact avec les limons de l'unité 6.2.6, est marqué par une légère décarbonatation des éléments. La stratification, faiblement exprimée, est subhorizontale. Bien représentés dans les environs de la ferme de la Beuchille, les graviers de l'unité 9, épais de $1.5 \mathrm{~m}$ au maximum, se biseautent progressivement vers l'est où ils finissent par disparaître totalement.

\subsection{2 - Unité 6.2.6}

Cette unité consiste en plusieurs couches limoneuses à la base desquelles sont intercalées des lentilles graveleuses et sableuses. Ces lentilles parlent clairement en faveur d'une genèse fluviatile (limons d'inondation, alluvions distales). Au sommet, par contre, la texture est franchement silteuse, proche des dépôts lœssiques rencontrés en Ajoie, au nord du Jura plissé. Une hydromorphie très marquée confère à cette unité une teinte jaune orange. Plus épaisse à l'est, où elle atteint $2.5 \mathrm{~m}$ de puissance, elle a probablement érodé le sommet des graviers de la nappe T3. En revanche, à l'ouest, il semble qu'elle se soit déposée en continuité avec ces mêmes graviers. La partie sommitale de l'unité 6.2.6, très compacte et à granulométrie silteuse, est localement traversée par des fentes grises déferrifiées sur une épaisseur de 50 à $80 \mathrm{~cm}$. En section horizontale, celles-ci dessinent un réseau polygonal interprété comme un horizon structural fortement compacté, de type fragipan (fig. 7). On en déduit qu'à une phase de sédimentation fluviatile a succédé une phase à composante éolienne de plus en plus dominante. Ces sédiments lœssiques, très sensibles au gel, auraient favorisé la genèse d'un fragipan à leur sommet vers la fin du Pléniglaciaire supérieur würmien: les formations tardiglaciaires qui coiffent T2 ne montrent effectivement jamais de telles structures de gel.

\subsection{3 - Unités 6.2.5, 6.1 et 2}

L'unité 6.2.6 est recouverte tantôt par des limons d'inondation, sableux à la base et rapportés au Tardiglaciaire (unité 6.2.5), tantôt par des colluvions d'âge protohistorique (unité 6.1) ou historique (unité 2), qui peuvent ensemble atteindre $1 \mathrm{~m}$ d'épaisseur au maximum. Dans les trois cas, la base de ces unités est érosive, ce qui explique la présence discontinue du fragipan qui caractérise normalement le sommet de l'unité 6.2.6.

\subsection{4 - Stratigraphie du sondage OSL 1}

La géométrie des dépôts une fois connue, un sondage supplémentaire a été ouvert sur le plateau de la Beuchille dans un secteur présentant un développement optimal des couches, dont l'épaisseur totale dépasse $3 \mathrm{~m}$ (sondage OSL 1, fig. 2). La stratigraphie rencontrée est la suivante (fig. 5):

- Couche 1: Silts limoneux brun foncé, humifères. Horizon agricole actuel.

- Couche 2: Silts sablonneux brun-jaune clair, de consistance ferme, bioturbé, légèrement grumeleux et contenant quelques gravillons siliceux. Limite inférieure nette et ondulante, probablement érosive. Colluvions.

- Couche 6.2.6.1 : Silts limoneux brun rouille tachetés de gris, de consistance très ferme, très oxydés (Fe, Mn). Structure polyédrique à tendance lamellaire. Présence de zones grises déferrifiées qui forment des fentes de consistance moins ferme. Limite inférieure graduelle. Horizon fragipan développé sur des sédiments d'origine éolienne.

- Couche 6.2.6.2: Silts limoneux brun clair rouille tachetés de gris, de consistance très ferme, oxydés ( $\mathrm{Fe}$, $\mathrm{Mn})$. Structure à tendance polyédrique. Présence de fissures grises très nettes qui, en plan, dessinent un réseau polygonal bien marqué. Limite inférieure diffuse. 


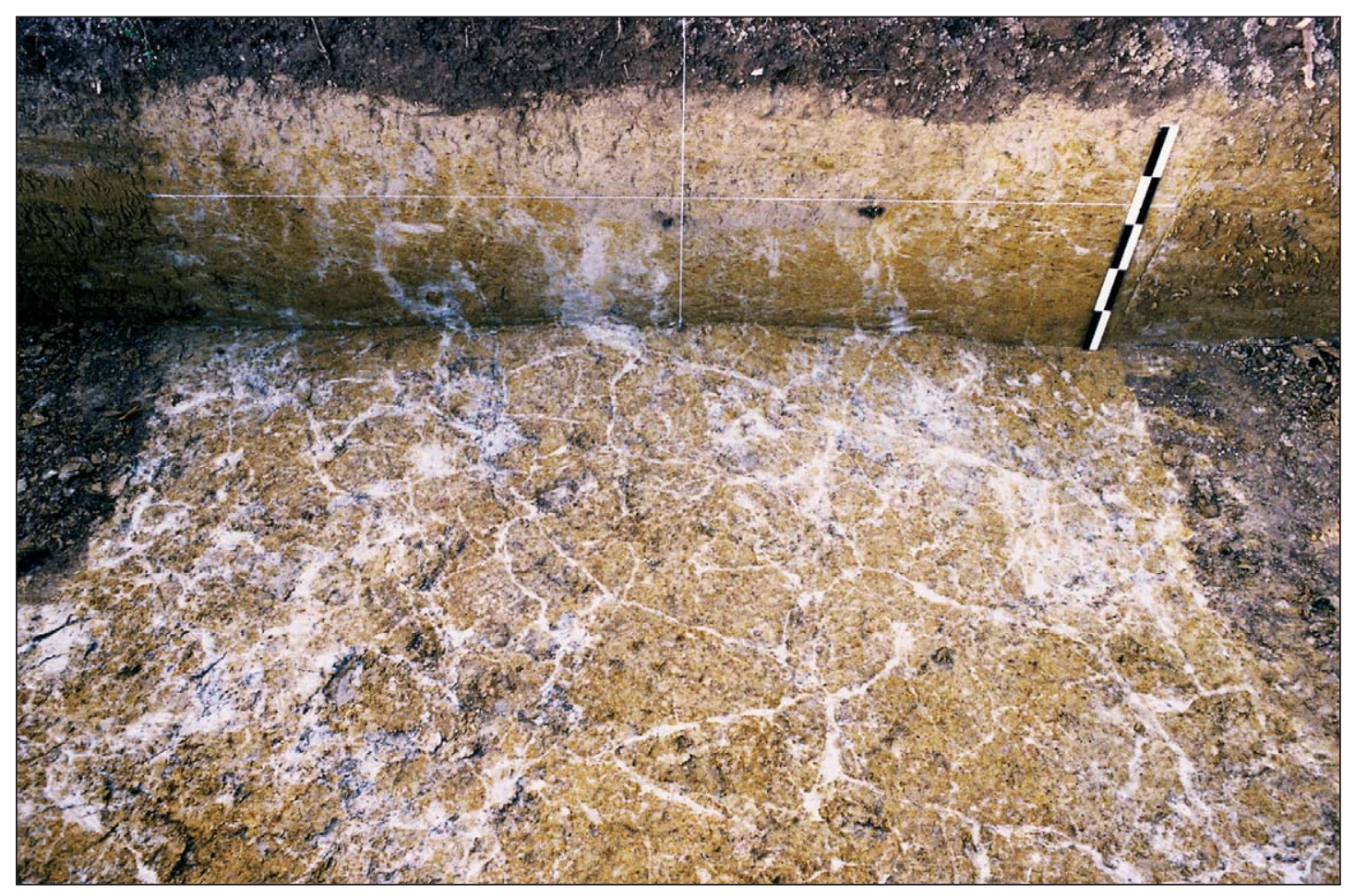

Fig. 7 : Polygones de gel typiques du fragipan développé dans la couverture loessique de la terrasse T3, vus en plan et en coupe (le jalon représente $50 \mathrm{~cm}$ ).

Fig. 7: Frost polygons, developed in the loessic cover from terrace T3, seen in cross-section and plan view (the scale represents $50 \mathrm{~cm}$ ).

Horizon fragipan développé sur des sédiments d'origine éolienne.

- Couche 6.2.6.3: Limons argileux brun clair rouille, de consistance ferme, contenant de rares gravillons siliceux; structure massive, absence de litage. Encroûtements manganiques en feuillets ou nodules. Limite inférieure diffuse. Limons d'inondation.

- Couche 6.2.6.4: Limons brun clair jaunâtre, de consistance moyenne à molle, légèrement sableux, micacés; structure massive. Limite inférieure abrupte. Limons d'inondation.

- Couche 9: Graviers calcaires arrondis, mal classés, dans une matrice limoneuse grise. Rares éléments siliceux $(<10 \%)$. Diamètre moyen des éléments: $1-3 \mathrm{~cm}$; diamètre maximal: $15 \mathrm{~cm}$. Stratification subhorizontale discrète. Limite inférieure érosive, légère décarbonatation sur $10 \mathrm{~cm}$ d'épaisseur au sommet. Graviers fluviatiles.

- Molasse: marne grise.

\section{2 - DONNÉES ANALYTIQUES}

\subsection{1 - Granulométrie}

Le diagramme So/Md (fig. 8), sur lequel ont été reportés les paramètres granulométriques de 13 échantillons prélevés dans différents profils de l'unité 6.2.6, montre deux nuages de points distincts. Le premier groupe, relativement mal défini, concerne les alluvions fines et limons d'inondation recouvrant les graviers de la nappe T3 (unité 6.2.6 base, lentilles graveleuses exceptées). Le second, très bien défini, se rapporte aux sédi- ments silteux de la partie sommitale de l'unité 6.2.6, interprétés comme des lœss. Les caractéristiques granulométriques de ces derniers, à savoir un indice de classement (So) de 2.5-3, une médiane (Md) de 12-15 microns et un coefficient d'asymétrie ( $\mathrm{Sk}$ ) de $0.8-0.95$, sont identiques à celles des loess rencontrés en Ajoie (So : 2-3; Md: 10-20 microns; Sk: 0.8-0.95, Aubry et al., 2000; Braillard, 2006), confirmant par la même le diagnostic de terrain.

On notera encore, sur le diagramme So/Md, la proximité des points se rapportant aux graviers T2 (So: 3.5 ; Md: $2.2 \mathrm{~cm}$ ) et T3 (So: $4.2 ; \mathrm{Md}: 1.05 \mathrm{~cm}$ ), ce qui suggère des conditions de dépôt similaires pour ces deux niveaux de cailloutis.

\subsection{2 - Géochimie}

De manière générale, les paramètres géochimiques varient peu, aussi bien verticalement le long d'un même profil qu'entre les différentes stratigraphies étudiées de la nappe T3.

Si l'on excepte les passées graveleuses qui marquent sa base, l'unité 6.2.6 est totalement décarbonatée. Il en va de même pour les unités $6.2 .5,6.1$ et 2. La matrice des graviers de l'unité 9 comprend quant à elle environ 30\% de carbonates. Les valeurs de $\mathrm{pH}$, faiblement acides, oscillent généralement entre 5 et 6.5 , même si quelques échantillons indiquent un $\mathrm{pH}$ extrême de 3.6. Au contact avec le cailloutis de base, le pH devient neutre (6.5 - 7.5). Le taux d'humus est nul dans les couches des unités 9 , 6.2.6 et 6.2.5, alors qu'il est relativement élevé (0.02 0.35 u.c.) dans les colluvions protohistoriques et historiques. Les teneurs en phosphates sont pour ainsi dire 


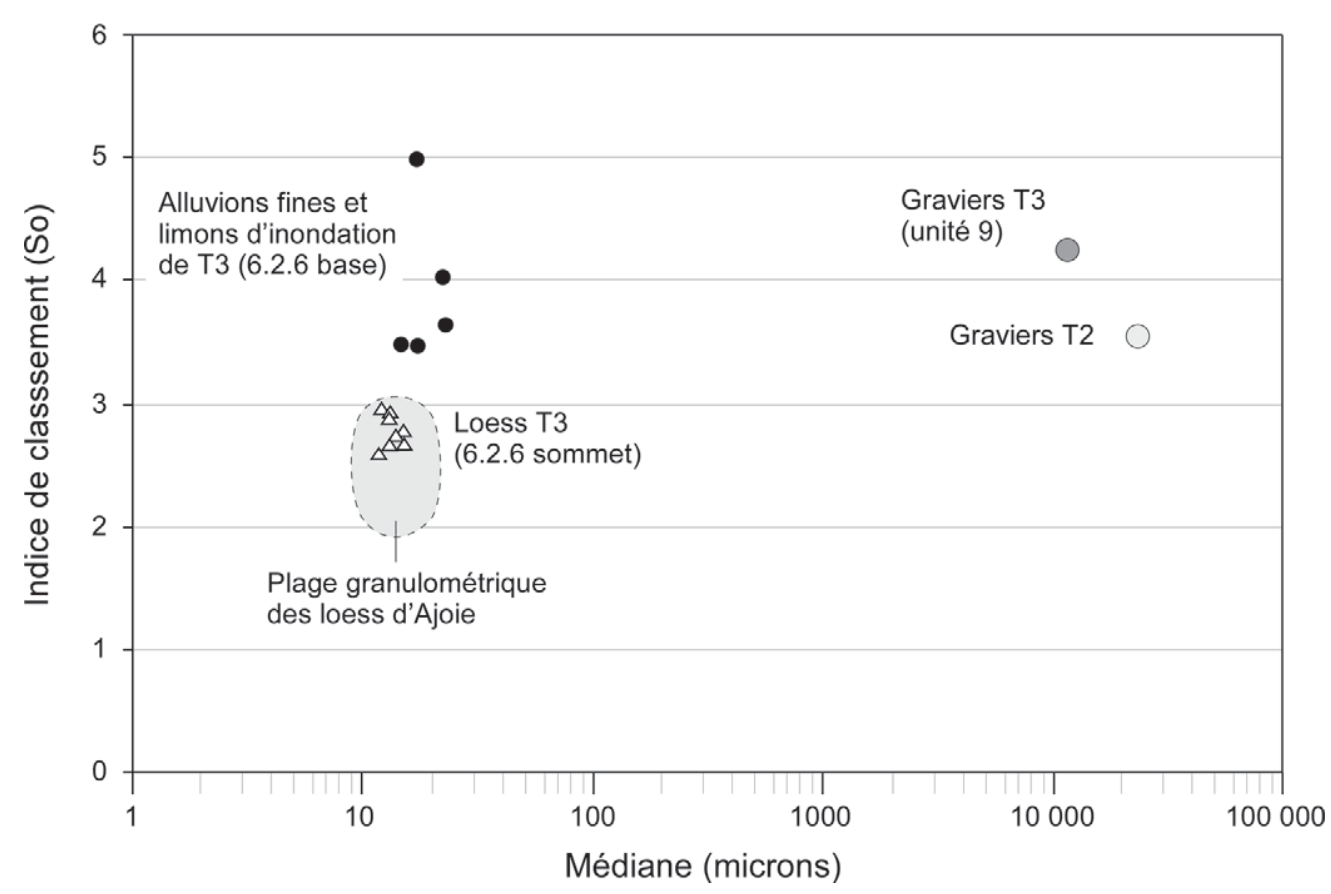

Fig. 8: Paramètres granulométriques des unités de T3, des graviers de T2 et des lœss d'Ajoie.

Fig. 8: Grain-size parameters of T3 and T2 gravel units, and Ajoie loess.

nulles dans toutes les couches et la teneur en matière organique n'est significative que dans l'horizon agricole.

Bien qu'ils suggèrent qu'une longue évolution pédologique, à savoir décarbonatation, brunification et forte hydromorphie, a affecté l'unité 6.2.6, ces tests géochimiques n'ont mis en évidence aucun véritable paléosol dans la séquence, que cela soit au sein des alluvions inférieures que dans les dépôts lœssiques supérieurs.

\subsection{3 - Microscopie}

L'analyse de lames minces sous microscope optique a porté essentiellement sur la nature et l'origine des sédiments du sommet de l'ensemble 6.2.6, interprétés comme des loss. L'assemblage minéralogique (quartz dominants, micas clairs, rares feldspaths et amphiboles) la forme des grains (anguleux à subanguleux), l'excellent tri des particules ainsi que la disposition aléatoire des micas sont autant de critères également reconnus dans les loess d'Ajoie. Cette similitude montre que le sommet de la nappe alluviale $\mathrm{T} 3$ est effectivement constitué d'apports éoliens allochtones.

\section{3 - DATATIONS OSL}

Les sédiments constituant la nappe alluviale T3 étant dépourvus de matière organique et des tests palynologiques s'étant révélés négatifs, nous avons entrepris de la dater par luminescence stimulée optiquement (OSL).

Le choix des sédiments a porté sur les limons qui recouvrent les graviers de T3 (couches 6.2.6.3 et 6.2.6.4, fig. 5). L'horizon lœssique à fragipan (couches 6.2.6.1 et 6.2.6.2) n'a en revanche pas été retenu, à cause de son hétérogénéité (fissures de gel) et de sa relative pauvreté en sables, mais aussi du fait de sa consistance très ferme rendant tout prélèvement difficile. Pour ces mêmes raisons techniques, mais également pour éviter une possible surestimation de l'âge liée à un éventuel blanchiment incomplet des grains, les graviers (unité 9) n'ont pas été prélevés.

L'échantillon inférieur (LB 3, tab. 1) a livré un âge de $39.4 \pm 6.8$ ka, l'échantillon médian (LB 2) $26.7 \pm 4.0 \mathrm{ka}$ et l'échantillon supérieur (LB 1) $21.8 \pm 2.8 \mathrm{ka}$. Conformes à l'empilement stratigraphique, ces datations

\begin{tabular}{|c|c|c|c|c|c|c|c|c|c|}
\hline $\begin{array}{c}\text { Code } \\
\text { laboratoire }\end{array}$ & Echantillons & $\begin{array}{c}\text { Teneur en } \\
\text { eau actuelle } \\
(\%)\end{array}$ & $\begin{array}{c}\text { Uranium } \\
(\mathrm{ppm})\end{array}$ & $\begin{array}{c}\text { Thorium } \\
\text { (ppm) }\end{array}$ & $\begin{array}{c}\text { Potassium } \\
(\%)\end{array}$ & $\begin{array}{l}\text { alpha- } \\
\text { efficiency } \\
\text { factor }\end{array}$ & $\begin{array}{c}\text { Dose } \\
\text { annuelle } \\
\text { (Gy ka-1) }\end{array}$ & $\begin{array}{l}\text { Paléodose } \\
\text { (Gy) } \\
\text { MAR (IRSL) }\end{array}$ & Age (ka) \\
\hline C-L1052 & DEL-LB 1 & 20 & $2,08 \pm 0,17$ & $13,4 \pm 0,7$ & $1,83 \pm 0,09$ & $0,09 \pm 0,03$ & $3,91 \pm 0,32$ & $85 \pm 9$ & $21,8 \pm 2,8$ \\
\hline C-L1053 & DEL-LB 2 & 20 & $2,31 \pm 0,18$ & $13,9 \pm 0,7$ & $1,81 \pm 0,09$ & $0,09 \pm 0,03$ & $4,03 \pm 0,34$ & $108 \pm 14$ & $26,7 \pm 4,0$ \\
\hline C-L1054 & DEL-LB 3 & 20 & $2,54 \pm 0,15$ & $13,3 \pm 0,7$ & $2,02 \pm 0,10$ & $0,09 \pm 0,03$ & $4,23 \pm 0,34$ & $166 \pm 26$ & $39,4 \pm 6,8$ \\
\hline
\end{tabular}

Tab. 1 : Paramètres des datations OSL (MAR: Multiple Aliquots Regenerative dose protocol; IRSL: Infrared stimulated luminescence). Tab. 1: OSL parameters (MAR: Multiple Aliquots Regenerative dose protocol; IRSL: Infrared stimulated luminescence). 
indiquent une mise en place des dépôts de l'unité 6.2.6base entre la seconde partie du Pléniglaciaire moyen et le Pléniglaciaire supérieur würmiens.

\section{4 - DISCUSSION}

Les caractéristiques sédimentologiques des graviers de la nappe alluviale T3 (classement médiocre, éléments grossiers) indiquent une mise en place générée par des courants très rapides dans un système à chenaux multiples, de type tressage. Il en va de même pour les graviers de la nappe alluviale T2, dont l'épaisseur est cependant nettement plus conséquente $(6 \mathrm{~m}$ au maximum et $1,5 \mathrm{~m}$ seulement pour T3). Cette dynamique active nécessite des flux hydriques importants, issus de tout le bassin versant. En revanche, la séquence limoneuse qui coiffe le cailloutis T3 marque un changement drastique de la sédimentation: ces dépôts s'apparentent à des limons d'inondation déposés par des courants beaucoup plus lents. Bien que comportant encore, à la base, quelques passées sableuses et gravillonneuses qui traduisent des épisodes hydrologiquement un peu plus énergiques, ils impliquent un transport et des flux nettement moins importants que pour l'unité sous-jacente. Cette formation provient ainsi de processus discontinus qui remanient des terrains quaternaires (altérites, éboulis) et molassiques (marnes et grès) plus anciens affleurant sur le flanc nord du Mont Chaibeux. Elle est coiffée par des sédiments lœssiques pédogénisés. Sommairement, on peut donc dire que la séquence recouvrant les graviers de T3 est issue d'apports locaux fins, générés à la base par du ruissellement épisodique, et qui vers le sommet deviennent progressivement de plus en plus allochtones, issus d'un transport par voie éolienne.

Les datations OSL de la partie inférieure de ces dépôts présentent une fourchette chronologique assez large: de 19 à 46 ka en tenant compte des marges d'erreurs. Se rapportant à une épaisseur de $30 \mathrm{~cm}$ de sédiments, ceci atteste du caractère discontinu de la sédimentation. Une telle succession, dans laquelle se marque aussi une pédogenèse peu évoluée, évoque en outre les dépôts limoneux mis en place au cours du Pléniglaciaire moyen würmien, comme nous avons pu les identifier au nord du Jura plissé, en Ajoie (Guélat, 2000).

Par conséquent, l'âge des graviers de la nappe T3 est plus ancien que cet intervalle. Aussi bien la géométrie des couches que des arguments pédostratigraphiques semblent montrer qu'il existe une continuité entre le cailloutis et la base de la séquence limono-sableuse, le contact entre les deux unités étant dans ce cas de type sédimentaire. Active au cours des arrêts qui ont jalonné l'édification de la nappe alluviale, la décarbonatation a touché le sommet des graviers, sans toutefois atteindre un degré très avancé. Selon ce scénario, les graviers de T3 se seraient déposés dans une période allant du Pléniglaciaire inférieur au début Pléniglaciaire moyen. Cette option a l'avantage de s'accorder avec le schéma climatique de genèse des terrasses fluviatiles en moyenne montagne, les dépôts grossiers étant l'expression des stades les plus froids (Léger, 1984). Cependant, nous ne pouvons exclure que ce contact soit de type érosif. Dans ce cas, la frange altérée des graviers résulterait d'une phase de pédogenèse antérieure au Pléniglaciaire moyen. On pourrait ainsi étendre la période possible du dépôt de ces graviers jusqu'au Glaciaire ancien (SI 5a-d, environ 75 à $115 \mathrm{ka} \mathrm{BP}$ ).

A partir des considérations qui précèdent, une réflexion peut être formulée à propos de la phase de creusement ayant mené à l'étagement de T3 par rapport à T2. A l'évidence, celle-ci a eu lieu après l'édification du cailloutis de T3, parallèlement à la genèse de la séquence limoneuse: le changement de régime sédimentaire décrit plus haut témoignerait ainsi du début de cette incision qui s'insérerait entre la seconde partie du Pléniglaciaire moyen (soit entre 45 et $30 \mathrm{ka}$ environ) et le début du Pléniglaciaire supérieur (soit avant 25 ka environ) durant lequel s'est accumulé le cailloutis T2. L'enfoncement de T2 par rapport à T3 atteignant $18 \mathrm{~m}$ environ à l'aplomb du profil daté, on obtient un taux d'incision compris entre 0,9 et $3,6 \mathrm{~mm} / \mathrm{an}$. Ces valeurs nous paraissent plutôt élevées, mais elles peuvent s'expliquer par le contexte particulier du secteur étudié. D'une part, il se situe en bordure de glaciers locaux qui recouvraient les sommets jurassiens au cours des phases froides du Würm et à une vingtaine de kilomètres seulement du bord de l'inlandsis alpin, dont une partie des eaux de fonte s'écoulait peut-être vers le nord via les cluses ou le karst (fig. 3). D'autre part, il se trouve dans une zone de confluence des cours d'eau principaux (fig. 2). On peut par conséquent imaginer que les flux hydriques furent, de manière récurrente, très importants au centre du bassin de Delémont et purent de ce fait engendrer une force érosive conséquente.

La présente étude révèle également que des apports éoliens allochtones coiffent la nappe alluviale T3. Plusieurs critères analytiques (cortège minéralogique, forme et tri des grains, texture) tendent à montrer que ceux-ci s'apparentent aux loss identifiés en Ajoie. A priori, on peut donc penser que les dépôts lœssiques du plateau de la Beuchille ont une origine identique à ces derniers, à savoir la plaine alluviale du Rhin (Aubry et $a l ., 2000)$, éloignée d'une trentaine de kilomètres de la vallée de Delémont. On relèvera toutefois que le substrat molassique de ce synclinal peut présenter, selon le faciès, un assemblage minéralogique similaire, mais que ses caractéristiques granulométriques diffèrent passablement. Chronostratigraphiquement, les lœss du bassin de Delémont s'insèrent à la fin du Pléniglaciaire supérieur, soit après 20 ka selon les datations OSL, et les polygones de gel s'y sont développés après leur mise en place. Ces figures cryogéniques s'expliquent par la présence d'un pergélisol, dont la trace est enregistrée sous la forme d'un horizon fragipan. Sa genèse se place habituellement au début du Tardiglaciaire dans le domaine périglaciaire de l'ouest européen (Van Vliet-Lanoë et al., 1992). 


\section{5 - CONCLUSIONS}

La nappe alluviale T3 ou «haute terrasse» du bassin de Delémont constitue le reflet, bien que lacunaire, d'événements pédosédimentaires majeurs ayant eu lieu dans les vallées jurassiennes demeurées libres de glace durant la dernière glaciation.

La dynamique sédimentaire très énergique qui a conduit à l'édification de son cailloutis de base s'inscrit dans un contexte paléoclimatique froid. Selon les différents arguments chronologiques et stratigraphiques explicités dans le présent article, il s'agirait préférentiellement du Pléniglaciaire inférieur würmien. La base de la séquence limoneuse accumulée au-dessus de ces graviers se rapporte, comme l'indiquent les datations OSL, au Pléniglaciaire moyen. Caractérisée par une relative décrue glaciaire sur le Plateau suisse (Graf, 2006), cette période voit également l'enfoncement assez conséquent du fond de la vallée. Le contexte local, à savoir une faible distance par rapport aux zones englacées et la conjonction de bassins versants régionaux, pourrait expliquer que cette incision s'est effectuée à un taux relativement élevé, de l'ordre du millimètre/an. Une implication du facteur néotectonique n'est toutefois pas à exclure.

Ces résultats surprennent compte tenu de la différence d'altitude, de l'ordre d'une vingtaine de mètres, observée entre la nappe T3 et celle du Pléniglaciaire supérieur, ou $\mathrm{T} 2$ : on pouvait en effet s'attendre à un âge beaucoup plus grand, à savoir anté-éémien ou plus ancien, comme les travaux précédents l'avaient suggéré. Ces nouvelles données mettent ainsi en évidence l'importance des informations lithostratigraphiques et paléopédologiques collectées à partir de profils ouverts au sein même des nappes alluviales. Idéalement, celles-ci doivent être appuyées par des datations issues de méthodes physiques telles que l'OSL.

Un autre acquis de cette étude réside dans l'identification formelle de dépôts éoliens dans une vallée du Jura plissé. Ils s'insèrent, toujours selon les datations par luminescence, dans la seconde partie du Pléniglaciaire supérieur. Apparentés à ceux d'Ajoie (pied nord du Jura), ces lœss n'apparaissent que sporadiquement dans la vallée de Delémont. Le site de la Beuchille est en effet la seule station, sur $15 \mathrm{~km}$ de tracé autoroutier prospecté, où de tels dépôts ont pu être observés, avec en plus l'apparition conjointe de polygones de gel appartenant à un horizon fragipan. Ce dernier traduit la présence locale, vers la fin du Pléniglaciaire supérieur, d'un pergélisol au sommet de la terrasse T3.

En ce qui concerne les autres nappes alluviales étagées, à savoir $\mathrm{T} 2$ et $\mathrm{T} 1$, les études liées à l'autoroute A16 en cours dans la vallée de Delémont apporteront des compléments d'informations indispensables quant aux conditions et à la chronologie de leur édification. A ce moment-là pourra être accomplie une approche intégrale de ce système témoignant du dernier cycle glaciaire dans les vallées intrajurassiennes.

\section{REMERCIEMENTS}

Cette étude a été rendue possible grâce au soutien de l'Office de la culture du canton du Jura. Nous remercions Alexandra Hilgers, du Département de Géographie de l'Université de Cologne (D), qui a réalisé les datations OSL. Les analyses granulométriques et géochimiques ont été effectuées au Laboratoire de l'IPNA (Institut für Prähistorische und Naturwissenschaftliche Archäologie) de l'Université de Bâle par Beatrix Ritter, à qui nous sommes reconnaissants. Merci encore à Thomas Beckmann (laboratoire indépendant à Braunschweig) qui s'est chargé de la fabrication des lames minces, ainsi qu'à Gérard Nicoud et Anne-Véronique WalterSimonnet, dont les commentaires constructifs ont amélioré le manuscrit.

\section{RÉFÉRENCES BIBLIOGRAPHIQUES}

ANTOINE P., ROUSSEAU D.-D., HATTÉ C., ZÖLLER L., LANG A., FONTUGNE M., \& MOINE O., 2002 - Evénements éoliens rapides dans les lœss du Pléniglaciaire supérieur weichsélien: l'exemple de la séquence de Nussloch (Vallée du Rhin-Allemagne). Quaternaire, 13 (3-4), 199-208.

AUBRY D., GUÉLAT M., DETREY J., OTHENIN-GIRARD B., et al., 2000 - Dernier cycle glaciaire et occupations paléolithiques à Alle, Noir Bois (Jura, Suisse). Cahier d'archéologie jurassienne, 10, Office de la culture et Société jurassienne d'Emulation, Porrentruy, $176 \mathrm{p}$.

AUBRY D., BRAILLARD L., GUÉLAT M., STALDER L., \& STAHL GRETSCH L.-I., 2005 - L'homme et l'environnement. Archéologie suisse, 28 (2), 6-16.

BARSCH D., 1969 - Studien zur Geomorphogenese des zentralen Berner Juras. Basler Beiträge zur Geographie, 9, 221 p.

BRAILLARD L., 2006 - Morphogenèse des vallées sèches du Jura tabulaire d'Ajoie (Suisse) : rôle de la fracturation et études des remplissages quaternaires. Thèse de Doctorat, Université de Fribourg, Geofocus, 14, 224 p.

BRAILLARD L., à paraître - Géologie et environnement sédimentaire. In O. Wey, Occupations protohistoriques à Delémont-Sud (JUSuisse). Cahier d'archéologie jurassienne, Office de la culture et Société jurassienne d'Emulation, Porrentruy.

BRAILLARD L., \& GUÉLAT M., 2000 - Géologie. In P. Paupe, Sondages sur la section 7 de l'A16, Fouilles 1999. Archéologie et Transjurane, 72, 13-33. (inédit).

BRAILLARD L., \& GUÉLAT M., 2002 - Le plateau de la Beuchille: synthèse géologique. In P.-A Moeschler, Sondages des sections 7 et 8 de l'A16, Fouilles 2001. Archéologie et Transjurane, 94, 11-28. (inédit).

BRAILlaRd L., GUÉlat M., POUSAZ N., \& RACHOUDSCHNEIDER A.-M., 2002 - Interactions entre occupation humaine et milieux naturels dans le bassin de Delémont (Jura, Suisse) : l'âge du Bronze, un point de rupture manifeste dans la dynamique sédimentaire et l'évolution de la végétation. In $\mathrm{H}$. Richard. \& A. Vignot (dir.), Equilibres et ruptures dans les écosystèmes durant les 20 derniers millénaires en Europe de l'Ouest. Actes du colloque international de Besançon, septembre 2000. Presses Universitaires FrancComtoises, 213-221.

CAMPY M., \& ARN R., 1991 - The Jura glaciers: palaeogeography in the Würmian circum-Alpine zone. Boreas, 20, 17-27.

GRAF H. R., 2006 - Stratigraphie des Eiszeitalters in der Nordschweiz. Abstract, th $^{\text {th }}$ Swiss Geoscience Meeting, Bern, 84-85.

GREPPIN J.-B., 1855 - Notes géologiques sur les terrains modernes, quaternaires et tertiaires du Jura Bernois et en particulier du val de Delémont. Nouveaux mémoires de la Société Helvétique des Sciences Naturelles, 14, Berne, $71 \mathrm{p}$.

GUÉLAT M., 2000 - Approche micromorphologique. In D. Aubry, Dernier cycle glaciaire et occupations paléolithiques à Alle, Noir Bois (Jura, Suisse). Cahier d'archéologie jurassienne, 10, Office de la culture et Société jurassienne d'Emulation, Porrentruy, 61-80.

GUÉLAT M., 2005 - Etude géologique. In B. Othenin-Girard, Courrendlin, En Solé (Jura, Suisse). Habitats protohistoriques et structures de drainage de l'Antiquité au XX $X^{e} s$. Archéologie et Transjurane, 124, 11-18. (inédit). 
GUÉLAT M., 2006 a - Le Quaternaire dans le canton du Jura. Les témoins de la période géologique la plus récente et leur signification. Actes 2005 de la Société jurassienne d'Emulation, Porrentruy, 9-32.

GUÉLAT M., 2006 b - Etude géologique. In B. Othenin-Girard, Habitat de l'âge du Fer à Courrendlin, En Solé (Jura, Suisse). Archéologie et Transjurane, 134, 9-19. (inédit).

GUÉLAT M., à paraître - Stratigraphie et sédimentologie des dépôts. Évolution du paysage et paléohydrologie à l'époque protohistorique (2000 - 200 av. J.-C.). In N. Pousaz, Delémont, En La Pran (Jura Suisse) : environnement et premières occupations préhistoriques. Cahier d'archéologie jurassienne, 22, Office de la culture et Société jurassienne d'Emulation, Porrentruy.

GUÉLAT M., BROMBACHER C., OLIVE C., WICK L., RACHOUD-SCHNEIDER A.-M., et al., à paraître - DevelierCourtételle, un habitat rural mérovingien: Environnement et exploitation du terroir au début du Moyen Age. Cahier d'archéologie jurassienne, 16, Office de la culture et Société jurassienne d'Emulation, Porrentruy.

GUÉLAT M., RACHOUD-SCHNEIDER A.-M., ESCHENLOHR L., \& PAUPE P., 1993. - Archives palustres et vestiges de l'Age du Bronze entre Glovelier et Boécourt (JU, Suisse). Cahier d'archéologie jurassienne, 4, Office de la culture et Société jurassienne d'Emulation, Porrentruy, $184 \mathrm{p}$.

HAVLICEK E., \& GOBAT J.-M., 1996 - Les apports éoliens dans les sols du Jura. Etat des connaissances et nouvelles données en pâturages boisés. Etude et Gestion des sols, 3 (3), 167-178.

IVY-OCHS S., SCHÄFER J., KUBIK P.W., SYNAL H.-A., \& SCHLÜCHTER C., 2004 - Timing of deglaciation on the northern Alpine foreland (Switzerland). Eclogae geologicae Helvetiae, 97 (1), 47-55.

KELLER W.T., \& LINIGER H., 1930 - Feuille 1086 Delémont, Carte 1. Atlas géologique de la Suisse 1:25 000, Commission géologique de la Société helvétique des Sciences naturelles.
LÉGER M. (coord.), 1984 - Signification dynamique et climatique des formations et terrasses fluviatiles quaternaires. Bulletin de l'Association Française pour l'Etude du Quaternaire, 17-18-19, 193 p.

LINIGER H., 1925 - Geologie des Delsberger Beckens und der Umgebung von Movelier. Matériaux pour la carte géologique de la Suisse, Nouvelle série, 55 (IV). Commission géologique de la Société helvétique des Sciences naturelles, Berne, $71 \mathrm{p}$.

MONJUVENT G., \& NICOUD G., 2002 - Glaciations. In J.C. Miskowsky (dir.), Géologie de la Préhistoire: Méthodes, Techniques, Applications. GEOPRE, Paris, 105-117.

PFIRTER U., ANTENEN M., HECKENDORN W., BURKHALTER R. M., GÜRLER B., \& KREBS D., 1997 Feuille 1106 Moutier, Carte 96. Atlas géologique de la Suisse 1 : 25000 , Service hydrogéologique et géologique national, Berne.

POCHON M., 1973 - Apports allochtones dans les sols jurassiens (Jura vaudois et Jura neuchâtelois). Bulletin de la Société neuchâteloise des Sciences naturelles, 96, 135-147.

SOMMÉ J., LAUTRIDOU J.P., HEIM J., MAUCORPS J., PUISSÉGUR J.J., ROUSSEAU D.D., THÉVENIN A., \& VAN VLIET-LANOË B., 1986 - Le cycle climatique du Pléistocène supérieur dans les lœss d'Alsace à Achenheim. Bulletin de l'Association Française pour l'Etude du Quaternaire, 1 (2), 97-104.

VAN VLIET-LANOË B, FAGNART J, P, LANGOHR R., \& MUNAUT A., 1992 - Importance de la succession des phases écologiques anciennes et actuelles dans la différenciation des sols lessivés de la couverture lœssique d'Europe occidentale: argumentation stratigraphique et archéologique. Science du sol, 30 (2), 75-93.

WINTLE A.G., 1997. Luminescence dating: Laboratory procedures and protocols. Radiation Measurements 27 (5/6), 769-817.

ZOLLINGER G., 1991. - Zur Landschaftsgenese und Quartärstratigraphie am südlichen Oberrheingraben - am Beispiel der Lössdeckschichten der Ziegelei in Allschwil (Kanton BaselLandschaft). Eclogae geologicae Helvetiae, 84 (3), 739-752. 